\title{
Correlates of the discrepancy between objective and subjective cognitive functioning in non-demented patients with Parkinson's disease
}

\author{
Mattia Siciliano $^{1,2} \cdot$ Lugi Trojano $^{2} \cdot$ Rosa De Micco $^{1} \cdot$ Valeria Sant'Elia $^{2} \cdot$ Alfonso Giordano $^{1}$ - Antonio Russo ${ }^{1}$. \\ Luca Passamonti ${ }^{3,4}$. Gioacchino Tedeschi ${ }^{1} \cdot$ Carlo Chiorri $^{5} \cdot$ Alessandro Tessitore $^{1}$
}

Received: 8 January 2021 / Revised: 8 March 2021 / Accepted: 10 March 2021 / Published online: 15 March 2021

(c) The Author(s) 2021

\begin{abstract}
Background Subjective complaints of cognitive deficits are not necessarily consistent with objective evidence of cognitive impairment in Parkinson's disease (PD). Here we examined the factors associated with the objective-subjective cognitive discrepancy.

Methods We consecutively enrolled 90 non-demented patients with PD who completed the Parkinson's Disease Cognitive Functional Rating Scale (subjective cognitive measure) and the Montreal Cognitive Assessment (MoCA; objective cognitive measure). The patients were classified as "Overestimators", "Accurate estimators", and "Underestimators" on the basis of the discrepancy between the objective vs. subjective cognitive measures. To identify the factors distinguishing these groups from each other, we used chi-square tests or one-way analyses of variance, completed by logistic and linear regression analyses. Results Forty-nine patients (54.45\%) were classified as "Accurate estimators", 29 (32.22\%) as "Underestimators", and 12 $(13.33 \%)$ as "Overestimators". Relative to the other groups, the "Underestimators" scored higher on the Fatigue Severity Scale (FSS), Beck Depression Inventory (BDI), and Parkinson Anxiety Scale $(p<0.01)$. Logistic regression confirmed that FSS and BDI scores distinguished the "Underestimators" group from the others $(p<0.05)$. Linear regression analyses also indicated that FSS and BDI scores positively related to objective-subjective cognitive discrepancy $(p<0.01)$. "Overestimators" scored lower than other groups on the MoCA's total score and attention and working memory subscores $(p<0.01)$. Conclusion In more than $45 \%$ of consecutive non-demented patients with PD, we found a 'mismatch' between objective and subjective measures of cognitive functioning. Such discrepancy, which was related to the presence of fatigue and depressive symptoms and frontal executive impairments, should be carefully evaluated in clinical setting.
\end{abstract}

Keywords Fatigue $\cdot$ Depression $\cdot$ Cognitive impairment $\cdot$ Subjective cognitive decline $\cdot$ Mild cognitive impairment

Luca Passamonti

1p337@medschl.cam.ac.uk

$\triangle$ Alessandro Tessitore

alessandro.tessitore@unicampania.it

1 Department of Advanced Medical and Surgical Sciences, MRI Research Center Vanvitelli-FISM, University of Campania “Luigi Vanvitelli”, Piazza Miraglia 2, 80138 Naples, Italy

2 Department of Psychology, University of Campania "Luigi Vanvitelli”, Viale Ellittico 31, 81100 Caserta, Italy

3 Department of Clinical Neurosciences, University of Cambridge, CB2 OSZ Cambridge, UK

4 Institute of Molecular Bioimaging and Physiology, CNR, Milan, Italy

5 Department of Educational Sciences, University of Genova, Genova, Italy

\section{Introduction}

Cognitive impairment is a frequent, pervasive and progressive non-motor manifestation of Parkinson's disease (PD) [1]. Considering the negative impact of cognitive deficits on patients' quality of life and functional independence [2], the International Parkinson and Movement Disorders Society (MDS) proposed consensus criteria for identifying mild cognitive impairment (MCI) in PD. This syndrome is characterized by objective cognitive deficits in addition to subjective complaints of cognitive impairments observed by either the patient, informant or clinician [1] and occurs in approximately $40 \%$ of $\mathrm{PD}$ population [3].

However, it is difficult to rely on patients' subjective reports of cognitive functioning, as around half of the 
patients with PD tend to judge their own cognitive performances as better or worse than their actual performances on objective cognitive testing [4].

Previous studies on the relationships between subjective cognitive complaints and objective cognitive impairment in PD led to conflicting results. Some authors did not observe statistically significant associations between subjective and objective cognitive functioning (e.g., Refs. [5, 6]). Others showed that subjective cognitive complaints tended to be more frequent in patients who later developed MCI or dementia (33-70\%), although the percentage of patients who complained about cognitive deficits but did not develop MCI or dementia was also high [7-14].

The opposite situation, i.e., a lack of subjective cognitive complaints in patients with objective cognitive impairments [4], has also been reported in PD. This 'cognitive anosognosia' is linked to disease progression and is tightly related to deficits in frontal lobe and executive functioning $[4,15]$.

As self-awareness of cognitive performance is also associated to motivation and emotional processing [16], the objective-subjective cognitive discrepancy in PD has been hypothesized to be moderated by psychiatric and behavioral symptoms [17].

To date, compelling evidence supports the idea that depression contributes to the objective-subjective cognitive discrepancy $[4,9,12,14,15,18-20]$. However, no conclusive evidence is available regarding the possible relationship with other common behavioral symptoms in PD (e.g., apathy, anxiety, fatigue, sleep disorders) or demographic and clinical features. For example, some studies showed that subjective cognitive complaints were more strongly related to anxiety [21] or fatigue [22] than to objective cognitive impairment, although other studies did not confirm this relationship [19]. These conflicting results may have stemmed from differences in the experimental designs and methodological approaches across studies, for instance the use of brief vs. more comprehensive behavioral evaluations. Moreover, only a few studies $[14,19]$ considered the potential impact of sleep disorders on the objective-subjective cognitive discrepancy. Yet evidence in healthy elderly [23] suggested that subjective cognitive measures were unlikely to provide accurate estimates of objective cognitive functioning in presence of sleep disturbances.

Therefore, a comprehensive evaluation of behavioral symptoms as well as of demographic and clinical features is necessary to identify the factors correlated with objectivesubjective cognitive discrepancy. Thus far, only two studies have used this approach and both reported a close relationship between depression and objective-subjective cognitive discrepancy. However, one study used a small sample size $(n=70 ;[19])$, while the other employed an informant-based behavioral scale $[4,24]$, that is known to potentially underestimate symptom severity [25].
To clarify the factors related to the objective-subjective cognitive discrepancy has implications for clinical practice and planning therapeutic strategies in PD. In clinical settings, where time resources are typically restricted, it is crucial to decide which depth of neuropsychological assessment is needed (i.e., level I screening vs. level II comprehensive evaluation; [1]). This clinical decision-making can be guided by the knowledge about the most important factors in determining cognitive deficits in PD, including the potential discrepancy between objective vs. subjective cognitive functioning.

In this study, we explored the main demographic, clinical, and patient-reported behavioral factors that were possibly associated to the objective-subjective cognitive discrepancy in a cohort of consecutive non-demented patients with PD.

Based on previous studies $[9,18,26]$, we expected that mood disturbances severity could increase the discrepancy between objective and subjective cognitive functioning.

\section{Methods}

\section{Patients and procedures}

One hundred eligible patients with a clinical diagnosis of idiopathic PD were consecutively screened at the Movement Disorders outpatient clinic of the First Division of Neurology, University of Campania "Luigi Vanvitelli" (Naples, Italy).

Exclusion criteria were: (1) history of cerebrovascular disorder or major and unstable medical disease; (2) lifetime or current psychotic disorders including major depressive episode, ascertained via the Mini International Neuropsychiatric Inventory [27]; (3) dementia, following the level I testing procedures proposed by MDS Task Force [28], in terms of co-occurrence of decreased global cognitive efficiency (i.e., age- and education-adjusted MoCA total score below Italian cut-off of 15.5 points; [29]), impairment in more than one cognitive domain (i.e., at least two age- and education-adjusted MoCA cognitive domain scores below Italian scores; [29]), and cognitive deficiency severe enough to impair daily life activities (based on medical records of patients' and caregivers' reports).

The local Ethical Committee supervised and approved all the procedures, following the Declaration of Helsinki. All participants gave their written informed consent before their inclusion in the study.

\section{Demographics and clinical features}

In all patients, we collected the following demographic characteristics: age, education, and sex. To assess the severity of motor symptoms, we used the motor section of the Unified 
Parkinson's Disease Rating Scale (UPDRS; [30]) and the Hoehn and Yahr staging system (HY; [31]). The patients were assessed in the "ON" state, and their medication regimen was recorded. Daily levodopa equivalent dosage $\left(\operatorname{LEDD}_{\mathrm{L}-\mathrm{DOPA}}\right)$, daily dopamine agonist equivalent dosage $\left(\operatorname{LEDD}_{\mathrm{DA}}\right)$, and the total amount of dopaminergic medication (LEDD total) were computed using Tomlinson et al.'s algorithm [32].

\section{Behavioral measures}

To characterize the behavioral profile of patients with PD, we used the Fatigue Severity Scale (FSS) [33, 34], the Beck Depression Inventory (BDI; [35]), the self-rated version of Parkinson Anxiety Scale [36, 37], and the self-rated version of Apathy Evaluation Scale (AES; [38, 39]). In addition, sleep problems were assessed via the Epworth Sleepiness Scale (ESS [40];) and the Parkinson's Disease Sleep Scale (PDSS; [41]).

\section{Cognitive assessment}

Objective cognitive functioning was assessed with the Montreal Cognitive Assessment (MoCA; [29, 42]), which provides a total score and six subscores for selected cognitive domains. The total scores were converted in age- and education-adjusted $Z$ scores (MoCA adjusted $Z$ scores; [29]). Higher MoCA adjusted $Z$ scores indicate better objective cognitive performance. The cut-off for the presence of objective cognitive impairment was a MoCA adjusted $Z$ score $\leq-1.5$.

Subjective cognitive complaints were assessed with the patient form of the Parkinson's Disease-Cognitive Functional Rating Scale (PD-CFRS; [43, 44]). This is a selfreport measure of cognitive dysfunction assessing the degree to which cognitive symptoms interfered with instrumental daily activities over the past 2 weeks. The PD-CFRS raw scores were converted in $Z$ scores [45], and then multiplied by -1 so to obtain $Z$ scores (PD-CFRS $Z$ score) with higher values indicating better subjective cognitive functioning. The cut-off for the presence of clinically significant subjective cognitive complaints was a PD-CFRS $Z$ score $\leq-1.5$.

The discrepancy between objective and subjective cognitive functioning was computed as the difference between the MoCA adjusted $Z$ scores and the PD-CFRS $Z$ scores.

On the basis of MoCA and PD-CFRS $Z$ score cut-offs, patients were categorized into three groups: "Underestimators", i.e., patients with subjective cognitive complaints but no objective cognitive impairment (i.e., PD-CFRS $Z$ score $\leq-1.5$ and MoCA adjusted $Z$ score $\geq-1.5$ ); "Accurate estimators", i.e., patients with neither subjective cognitive complaints nor objective cognitive impairment (i.e., PD-CFRS $Z$ score and MoCA adjusted $Z$ score $\geq-1.5$ ) or, alternatively, with both subjective cognitive complaints and objective cognitive impairment (i.e., PD-CFRS $Z$ score and MoCA adjusted $Z$ score $\leq-1.5$ ); "Overestimators", patients with objective cognitive impairment but no subjective cognitive complaints (i.e., PD-CFRS adjusted $Z$ score $\geq-1.5$ and MoCA adjusted $Z$ score $\leq-1.5$ ).

Our definition of "Underestimators" is similar to the one of "Subjective Cognitive Complaint" [14, 19] or "Subjective Cognitive Decline" $[13,46]$ which is often reported in the PD and Alzheimer's disease literature. However, here, we used the term "Underestimators" in an operative sense, without diagnostic implications. Likewise, our use of the term "Overestimators" might recall the concept of "cognitive anosognosia" [4] but also in this case we used the term "Overestimators" in an operational acceptation.

\section{Statistical analyses}

The data were tested for normality and values between -1 and 1 for asymmetry and kurtosis were considered acceptable.

Cohen's kappa $(\kappa)$ was calculated as a measure of agreement between the patients' subjective cognitive complaints (presence vs. absence) and objective evidence of cognitive impairment (presence vs. absence). The strength of agreement was interpreted as follows: $\kappa<0.00$ poor; $0.00 \leq \kappa \leq 0.20$ slight; $0.21 \leq \kappa \leq 0.40$ fair; $0.41 \leq \kappa \leq 0.60$ moderate; $0.61 \leq \kappa \leq 0.80$ substantial; $0.81 \leq \kappa \leq 1.00$ almost perfect [47].

We compared the groups of "Accurate estimators", "Underestimators", and "Overestimators" in terms of demographic, clinical, and behavioral features using Pearson's chi-square tests $(\chi 2)$ for categorical variables and one-way analyses of variance (ANOVA) for continuous variables. Pairwise post-hoc comparisons were used to determine which groups were significantly different and the directionality of the effects.

To identify the demographic, clinical, and behavioural features of the group of "Underestimators", we first carried out simple binary logistic regression analyses, to identify which features were able to discriminate the group of "Underestimators" from that of the "Non-underestimators" (i.e., "Overestimators" and "Accurate estimators" grouped together as in Ref. [48]), at a bivariate level. Second, we entered the features that showed a significant bivariate association with group membership in a multiple binary logistic regression analysis model (forced entry method) to test which ones independently contributed to explaining patient classification.

The associations between the demographic, clinical, and behavioral features and the objective-subjective cognitive discrepancy (difference between the MoCA $Z$ scores and 
PD-CFRS) was investigated using bivariate and multiple linear regression analyses (forced entry method).

To check the reliability of our findings, a bootstrap approach (1000 bootstrap) with a $95 \%$ bias corrected and accelerated confidence intervals $[95 \% \mathrm{CI}]$ was used. The bias of an estimate can be ignored if it is lower than 0.25 times its standard error [49].

Since the subjective cognitive complaints might have different implications in patients with or without objective cognitive impairment, we repeated all the analyses after excluding from the "Accurate estimators" group the patients with both subjective cognitive complaints and objective cognitive impairment.

All multiple comparisons were corrected for familywise errors by Bonferroni's procedures; Bonferroni corrected $p$ value $<0.05$ were considered statistically significant.

All statistics were performed using Statistical Package for Social Science version 20 (SPSS, Chicago, IL), All figures were created by GraphPad Prism 6.0 and Matlab.

\section{Results}

Out of 100 screened patients, eight were not willing to participate in our study, whereas two patients were excluded (of these one suffered from a current major depressive episode, and the other was diagnosed with PD-dementia).

The demographic and clinical features of included patients $(n=90)$ did not differ from those of screened patients who were not included $(n=10)$ (Supplementary Table 1).

The descriptive statistics of our final sample $(n=90)$ were reported in Table 1.

Asymmetry and kurtosis were acceptable for all continuous variables.

There was poor agreement between the patients' subjective cognitive complaints and objective evidence of cognitive impairment $(\kappa=-0.23, p=0.01)$.

Of the final sample, 49 patients $(54.45 \%)$ were classified as "Accurate estimators", 29 (32.22\%) as "Underestimators", and $12(13.33 \%)$ as "Overestimators" (Fig. 1a, b).

One-way ANOVAs and $\chi 2$ test, complemented by posthoc analyses, did not show statistically significant differences among "Overestimators", "Accurate estimators", and "Underestimators" in terms of demographic and clinical features. The group of "Underestimators" scored higher on the FSS, BDI, and PAS relative to the group of "Overestimators" and "Accurate estimators". The latter two groups did not differ between each other in terms of any behavioral feature considered. The group of "Underestimators" scored lower on the PD-CFRS than the group of "Overestimators" and "Accurate estimators". Relatively to the other groups, the group of "Overestimators" scored lower on the MoCA
Table 1 Overall sample descriptive statistics $(n=90)$

\begin{tabular}{|c|c|}
\hline Variable & Mean (SD) or count $(\%)$ \\
\hline \multicolumn{2}{|l|}{ Demographics } \\
\hline Age & $66.74(9.22)$ \\
\hline Education, years & $9.66(4.09)$ \\
\hline Sex, male & $53(58.90 \%)$ \\
\hline \multicolumn{2}{|l|}{ Clinical features } \\
\hline Age at onset & $61.63(9.72)$ \\
\hline Disease duration, years & $5.25(2.92)$ \\
\hline UPDRS-III & $27.33(9.57)$ \\
\hline Hoehn and Yahr stage & $2.00(0.37)$ \\
\hline LEDD total (mg/day) & $516.82(233.37)$ \\
\hline $\operatorname{LEDD}_{\mathrm{DA}}$ (mg/day) & $71.23(106.27)$ \\
\hline $\operatorname{LEDD}_{\text {L-DOPA }}(\mathrm{mg} /$ day $)$ & $386.16(259.29)$ \\
\hline \multicolumn{2}{|l|}{ Behavioural measures } \\
\hline Fatigue Severity Scale & $3.47(1.88)$ \\
\hline Beck Depression Inventory & $8.91(7.45)$ \\
\hline Parkinson Anxiety Scale & $11.92(9.26)$ \\
\hline Apathy Evaluation Scale & $31.74(7.55)$ \\
\hline Parkinson's disease sleep scale & $114.95(22.23)$ \\
\hline Epworth Sleepiness Scale & $5.77(4.19)$ \\
\hline \multicolumn{2}{|l|}{ Cognitive assessment } \\
\hline \multicolumn{2}{|l|}{ MoCA total } \\
\hline Raw score & $20.02(4.87)$ \\
\hline Adjusted score $^{\mathrm{a}}$ & $22.46(4.31)$ \\
\hline Adjusted $Z$ score $^{\mathrm{a}}$ & $0.18(1.42)$ \\
\hline \multicolumn{2}{|l|}{ MoCA adjusted subscores ${ }^{\mathrm{a}}$} \\
\hline Memory & $1.29(1.47)$ \\
\hline Visuospatial abilities & $0.68(1.40)$ \\
\hline Executive functions & $0.78(1.77)$ \\
\hline Attention, and WM & $5.01(1.03)$ \\
\hline Language & $3.22(1.52)$ \\
\hline Orientation & $5.76(0.57)$ \\
\hline \multicolumn{2}{|l|}{ PD-CFRS } \\
\hline Raw & $2.42(3.01)$ \\
\hline$Z$ score & $-0.68(2.32)$ \\
\hline $\begin{array}{l}\text { MoCA ( } Z \text { score) minus PD-CFRS ( } Z \\
\text { score) }\end{array}$ & $-0.64(2.30)$ \\
\hline
\end{tabular}

$S D$ standard deviation, UPDRS Unified Parkinson's Disease Rating Scale, $L E D D$ levodopa equivalent daily dose, $M o C A$ Montreal Cognitive Assessment, $W M$ working memory, PD-CFRS Parkinson's Disease Cognitive Functional Rating Scale

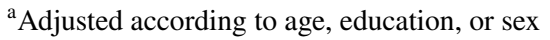

total score and the attention and working memory subscore of the MoCA (Table 2).

The simple binary logistic regression analyses showed that neither the demographic nor the clinical features could distinguish the group of "Underestimators" from that of the "Non-underestimators" (i.e., "Overestimators" or "Accurate estimators" grouped together as in Ref [48]). In contrast, the FSS, BDI, PAS, and AES were able to distinguish the 
Fig. 1 a Patients grouped according to the presence of objective cognitive impairment and subjective cognitive complaint. b Percentage of overestimators, accurate estimators, and underestimators (a)

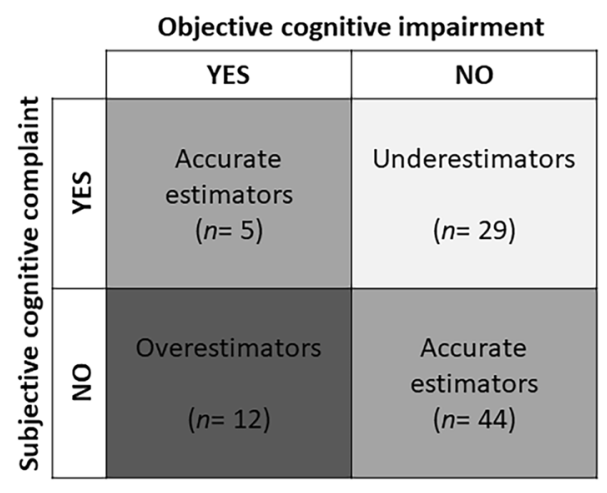

(b)

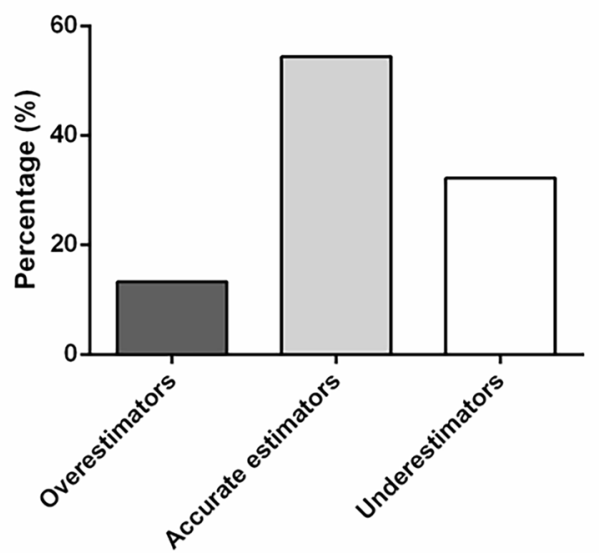

"Underestimators" from "Non-underestimators". However, the multiple binary logistic regression analysis revealed that only the BDI and FSS were independently able to classify the "Underestimators" vs. "Non-underestimators" with an overall accuracy of $76 \%$ (Table 3 ).

The bivariate linear regression analyses showed that higher scores on FSS, BDI, PAS, AES, and ESS were associated with a greater discrepancy between PD-CFRS $Z$ scores and the MoCA adjusted $Z$ scores. The multiple linear regression analysis indicated that only the FSS and BDI were significantly associated with a discrepancy between the MoCA $Z$ scores and the PD-CFRS $Z$ scores and with an overall model accuracy of $42 \%$ (Table 4; Fig. 2).

The results were confirmed after the exclusion of patients who showed a congruency between subjective cognitive complaints and objective cognitive impairment $(n=5)$ from the "Accurate estimators" group (Supplementary Tables 2, $3,4,5)$.

The bootstrapping procedure did not reveal substantial biases, and confirmed that our sample size was adequate to detect statistically meaningful effects [49].

\section{Discussion}

We studied the extent to which the objective-subjective cognitive discrepancy in PD was associated with demographic, clinical, and patient-reported behavioral features.

Three main results emerged. First, there was poor agreement between patient subjective reports and patient objective deficits, as $>45 \%$ of patients showed objective-subjective cognitive discrepancy. Second, the underestimation of cognitive performance in PD was associated with the severity of fatigue and depressive symptoms but not with the demographics or clinical features. This supports the idea that specific behavioral symptoms are the main correlates of objective-subjective cognitive discrepancy in PD [4, 14, 19, 21]. Third, the overestimation of cognitive performance was tightly associated with frontal executive impairments.

Our first main finding demonstrated that the objectivesubjective cognitive discrepancy is a remarkable clinical phenomenon in PD. Indeed, $32 \%$ of patients had subjective cognitive complaints but no objective cognitive impairment ("Underestimators"), while a 13\% showed an inverse pattern (i.e., objective cognitive impairment but no subjective cognitive complaint, "Overestimators"). These findings highlight that underestimation of one's own cognitive abilities is common and clinically relevant in PD [13, 14], consistent with a recent study, in which the objective-subjective cognitive discrepancy occurred in $45 \%$ of patients with PD, with a higher percentage (24\%) of patients underestimating their objective cognitive abilities and a lower percentage (21\%) overestimating it [4].

These results showed that not all PD patients with objective cognitive impairments are able to report their real cognitive performance, whereas not all PD patients reporting cognitive problems display 'objective' deficits [5]. As the clinical diagnosis of MCI in PD sometimes relies on cognitive impairments typically reported by the patient or informant [1], our findings suggest caution in relying on patients' subjective reports, especially in the absence of objective testing [6].

Our second main finding showed that severity of fatigue, depressive, and anxious symptoms distinguished "Underestimators" from "Overestimators" and "Accurate estimators". The link between cognitive underestimation and fatigue has been consistently demonstrated in patients with multiple sclerosis (e.g., Refs. [48, 50]). However, this association has been scarcely investigated in PD, and conflicting results have been reported [19, 22]. In PD, Kluger et al. [51] proposed that fatigue, defined as a significantly diminished level of energy or increased perception of effort 


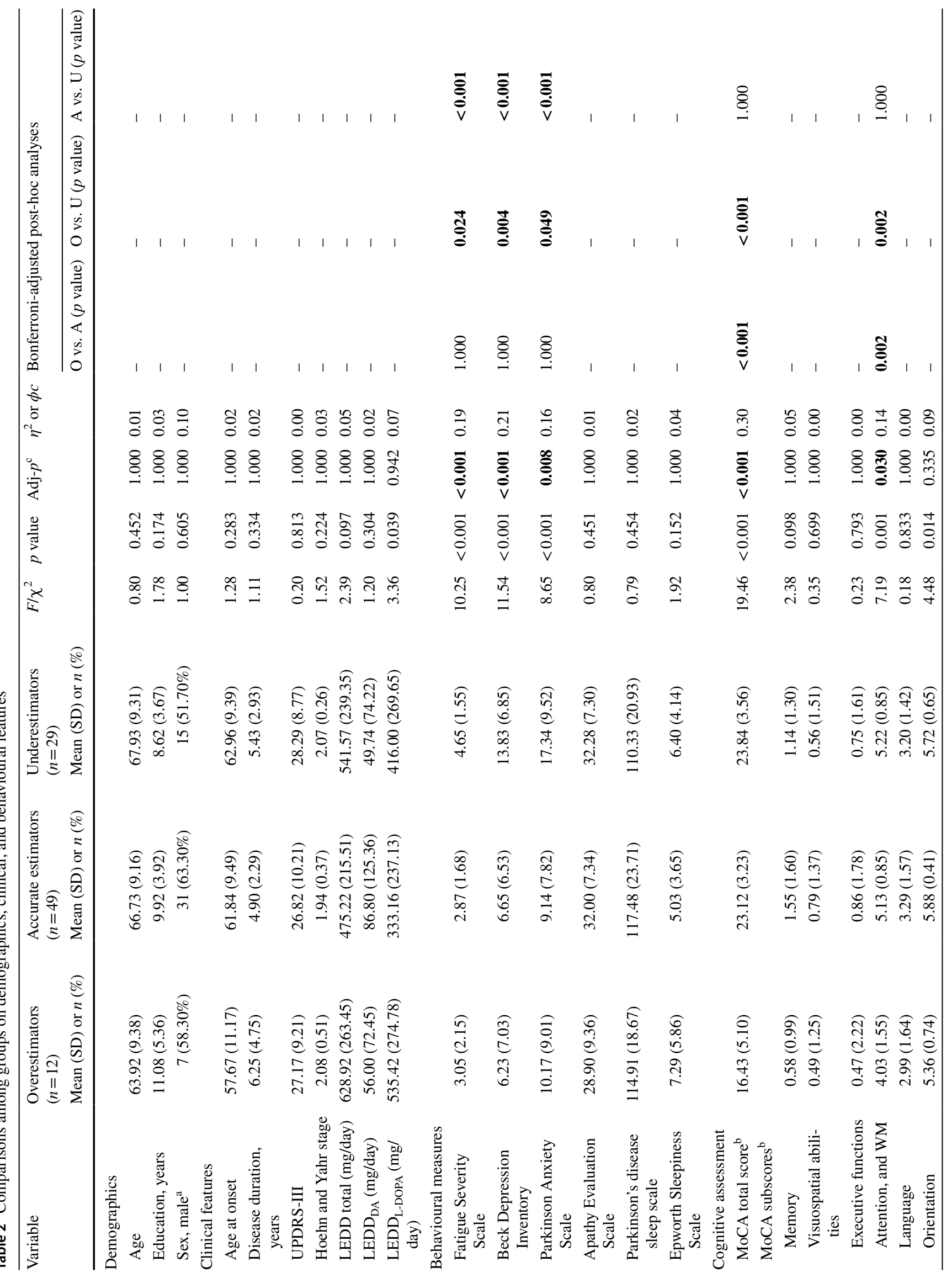


that is disproportionate to attempted activities [52], may be linked more to altered subjective awareness than to actual performance limitations. Indeed, fatigued patients often report subjective complaints of increased sense of effort or decreased stamina that are not related to objective decrement in performance [51]. In keeping with these findings, our group of "Underestimators" reported subjective complaints of cognitive deficits which were not supported by objective evidence of cognitive impairment.

Such link between fatigue and cognitive underestimation may stem, at least in part, from altered self-awareness (e.g., exaggeration of deficits or hyperawareness). According to Rosen's model [16], accurate self-awareness of performance depends on active monitoring of task performance, which results from comparing current performance with task demands and with the level of performance that is considered acceptable (e.g., a certain number of errors could be considered acceptable on a given task). Therefore, fatigue symptoms in "Underestimators" might be ascribed to alterations in self-awareness caused by inefficient monitoring of sensorimotor and cognitive processes. Recent evidence seems to support this speculation [53], but future studies are required.

"Underestimators" also showed more depressive and anxious symptoms than the two other groups, which is consistent with previous studies in PD $[9,12,14,18-20]$. Together, these findings demonstrate a tight association between selfawareness of cognitive performance and emotional processing [16]. Nevertheless, it remains unclear if mood symptoms alter self-awareness or whether a pre-existing deficit in self-awareness is antecedent to the development of mood symptoms [15]. It is also possible that more severe mood symptoms reported by the "Understimators" foster a negative bias in reporting problems or contribute to exaggerating the cognitive deficits (hyperawareness). This is because a negative and pessimistic vision of oneself, the environment, and the future is at the core of several mood disturbances [54].

Our third main finding revealed that, compared to the other groups, the "Overestimators" scored lower on the attention and working memory subscore of the MoCA (deriving from cognitive subtests such as Digit Span Backward [55], Serial 7 subtractions [56]). These results reinforce the evidence of an association between frontal executive impairments and poor self-awareness of cognitive deficits in PD [4, 15, 57], which is also in line with models of anosognosia in Alzheimer's disease [58].

Additional analyses corroborated our findings and confirmed their statistical robustness. First, we found that our main findings held when the "Overestimators" and "Accurate estimators" were merged in the same group ("Nonunderestimators") and compared to the group of "Underestimators" in logistic regression analyses. Second, we obtained 
Table 3 Simple and multiple binary logistic regression analyses assessing which demographics, clinical, and behavioural features distinguished underestimators from non-underestimators; $95 \%$ bias corrected and accelerated confidence intervals $[95 \% \mathrm{CI}]$ (1000 bootstrap samples) for the logistic regression coefficients were reported in parentheses

\begin{tabular}{|c|c|c|c|c|c|}
\hline Variable & Estimate [CI 95\%] & Bias & SE & $p$ value $^{\mathrm{b}}$ & OR [CI 95\%] \\
\hline \multicolumn{6}{|l|}{ Simple regression } \\
\hline \multicolumn{6}{|l|}{ Demographics } \\
\hline Age & $0.02[-0.03,0.07]$ & 0.00 & 0.02 & 0.399 & $1.02[0.97,1.07]$ \\
\hline Education, years & $-0.09[-0.22,0.01]$ & -0.00 & 0.06 & 0.101 & $0.90[0.80,1.01]$ \\
\hline $\operatorname{Sex}^{\mathrm{a}}$ & $-0.43[-1.29,0.50]$ & 0.04 & 0.45 & 0.342 & $0.64[0.26,1.58]$ \\
\hline \multicolumn{6}{|l|}{ Clinical features } \\
\hline Age at onset & $0.02[-0.02,0.07]$ & -0.00 & 0.02 & 0.379 & $1.02[0.97,1.07]$ \\
\hline Disease duration, years & $0.03[-0.13,0.20]$ & -0.00 & 0.08 & 0.691 & $1.03[0.88,1.19]$ \\
\hline UPDRS-III & $0.01[-0.02,0.06]$ & 0.00 & 0.02 & 0.520 & $1.01[0.96,1.06]$ \\
\hline Hoehn and Yahr stage & $0.80[-0.27,2.49]$ & 0.40 & 2.74 & 0.222 & $2.22[0.61,8.07]$ \\
\hline LEDD total (mg/day) & $0.00[-0.00,0.00]$ & 0.00 & 0.00 & 0.496 & $1.00[0.99,1.00]$ \\
\hline $\mathrm{LEDD}_{\mathrm{DA}}(\mathrm{mg} /$ day $)$ & $-0.00[-0.00,0.00]$ & 0.00 & 0.00 & 0.212 & $0.99[0.99,1.00]$ \\
\hline LEDD $_{\text {L-DOPA }}(\mathrm{mg} /$ day $)$ & $0.00[-0.00,0.00]$ & 0.00 & 0.00 & 0.471 & $1.00[0.99,1.00]$ \\
\hline \multicolumn{6}{|l|}{ Behavioural measures } \\
\hline Fatigue Severity Scale & $0.57[0.30,0.93]$ & 0.02 & 0.15 & $<0.001$ & $1.77[1.32,2.38]$ \\
\hline Beck Depression Inventory & $0.15[0.08,0.26]$ & 0.01 & 0.05 & $<0.001$ & $1.15[1.07,1.24]$ \\
\hline Parkinson Anxiety Scale & $0.10[0.05,0.16]$ & 0.00 & 0.02 & $<0.001$ & $1.10[1.04,1.16]$ \\
\hline Apathy Evaluation Scale & $0.10[0.03,0.19]$ & 0.00 & 0.04 & 0.024 & $1.10[1.01,1.20]$ \\
\hline Parkinson's disease sleep scale & $-0.01[-0.04,0.00]$ & -0.00 & 0.01 & 0.228 & $0.98[0.96,1.00]$ \\
\hline Epworth Sleepiness Scale & $0.05[-0.05,0.18]$ & 0.00 & 0.06 & 0.327 & $1.05[0.94,1.16]$ \\
\hline \multicolumn{6}{|l|}{ Multiple regression $^{c}$} \\
\hline Fatigue Severity Scale & $0.57[0.09,1.44]$ & 0.05 & 0.35 & 0.008 & $1.77[1.16,2.71]$ \\
\hline Beck Depression Inventory & $0.11[0.01,0.32]$ & 0.01 & 0.07 & 0.035 & $1.12[1.00,1.25]$ \\
\hline Parkinson Anxiety Scale & $0.01[-0.08,0.10]$ & 0.00 & 0.05 & 0.702 & $1.01[0.93,1.09]$ \\
\hline Apathy Evaluation Scale & $-0.06[-0.20,0.04]$ & -0.01 & 0.06 & 0.938 & $0.95[0.85,1.02]$ \\
\hline
\end{tabular}

Statistically significant variables are shown in bold

$S E$ standard error, $O R$ odds ratio, $C I$ confidence interval, UPDRS Unified Parkinson's Disease Rating Scale, $L E D D$ levodopa equivalent daily dose

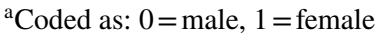

${ }^{\mathrm{b}} p$ value related to unstandardized beta coefficient using the Wald statistic

${ }^{\mathrm{c}}$ Model $\chi^{2}(4)=33.01, p$ value $<0.01, R^{2}=0.44$ (Nagelkerke) similar results when we explored the factors associated with objective-subjective cognitive discrepancy (i.e., the difference between the MoCA $Z$ scores and PD-CFRS) via the linear regression analyses in the whole patient sample.

When all the behavioural symptoms were evaluated simultaneously (by logistic or linear multiple regression analyses), depressive but not anxious symptoms were associated with the objective-subjective cognitive discrepancy. These results suggested that specific facets of depression, rather than those shared with anxiety (e.g., loss of energy) [59], may play a pivotal role in objective-subjective cognitive discrepancy. Interestingly, patients who are depressed, but not necessarily anxious, typically express negative beliefs about themselves (e.g., cognitive underestimation), the world, and the future [60].

All these findings highlight the importance of assessing and monitoring fatigue and depressive symptoms in PD, especially when patients' complaints of cognitive impairment are used as prognostic indicators of future objective cognitive deterioration (e.g., Refs. [10, 13, 14]).

We are aware that one limitation of the present study relates to the tools used to assess subjective cognitive complaints and objective cognitive impairment. As Jessen et al. [46] highlighted, a neuropsychological battery covering all domains is necessary for accurately evaluating the 'mismatch' between subjective and objective cognitive performance. The PD-CFRS has been validated as a measure of self-perceived impact of cognitive changes on daily functioning $[43,45]$, and provides only an indirect measure of subjective complaints of cognitive impairment. PD-CFRS is available in Italian and has been used in similar studies in PD [26], although it may be less specific than other scales (for a review, see Ref. [17]). The MoCA is a cognitive screening tool but is less sensitive to domain-specific dysfunctions than comprehensive neuropsychological batteries [1]. The use of MoCA and PD-CFRS may have partially 
Table 4 Simple and multiple linear regression analyses assessing which demographics, clinical, and behavioural features were associated with the discrepancy between objective and subjective cognitive functioning (MoCA $Z$ scores minus PD-CFRS $Z$ scores) in overall sample; $95 \%$ bias corrected and accelerated confidence intervals [95\% CI] (1000 bootstrap samples) for the linear regression coefficients were reported in parentheses

\begin{tabular}{|c|c|c|c|c|c|}
\hline Variable & Estimate [CI 95\%] & SE & Bias & $\beta$ & $p$ value \\
\hline \multicolumn{6}{|l|}{ Simple regression } \\
\hline \multicolumn{6}{|l|}{ Demographics } \\
\hline Age & $0.02[-0.03,0.07]$ & 0.02 & 0.00 & 0.07 & 0.468 \\
\hline Education, years & $-0.09[-0.18,0.04]$ & 0.05 & 0.00 & -0.14 & 0.186 \\
\hline $\operatorname{Sex}^{\mathrm{a}}$ & $-0.23[-1.26,0.74]$ & 0.52 & -0.00 & -0.04 & 0.674 \\
\hline \multicolumn{6}{|l|}{ Clinical features } \\
\hline Age at onset & $0.03[-0.02,0.09]$ & 0.02 & 0.00 & 0.12 & 0.236 \\
\hline Disease duration, years & $-0.15[-0.32,0.08]$ & 0.10 & 0.01 & -0.17 & 0.096 \\
\hline UPDRS-III & $0.00[-0.04,0.04]$ & 0.02 & 0.00 & -0.02 & 0.799 \\
\hline Hoehn and Yahr stage & $0.26[-0.80,1.33]$ & 0.53 & -0.02 & 0.03 & 0.721 \\
\hline LEDD total (mg/day) & $-0.00[-0.00,0.00]$ & 0.00 & 0.00 & -0.07 & 0.505 \\
\hline $\operatorname{LEDD}_{\mathrm{DA}}$ (mg/day) & $-0.00[-0.00,0.00]$ & 0.00 & 0.00 & -0.08 & 0.422 \\
\hline LEDD $_{\text {L-DOPA }}(\mathrm{mg} /$ day $)$ & $0.00[-0.00,0.00]$ & 0.00 & 0.00 & -0.04 & 0.714 \\
\hline \multicolumn{6}{|l|}{ Behavioural measures } \\
\hline Fatigue Severity Scale & $0.69[0.39,0.99]$ & 0.15 & -0.00 & 0.51 & $<0.001$ \\
\hline Beck Depression Inventory & $0.18[0.10,0.25]$ & 0.03 & 0.00 & 0.53 & $<0.001$ \\
\hline Parkinson Anxiety Scale & $0.11[0.05,0.16]$ & 0.02 & 0.00 & 0.40 & $<0.001$ \\
\hline Apathy Evaluation Scale & $0.09[0.03,0.16]$ & 0.03 & 0.00 & 0.27 & $\mathbf{0 . 0 1 0}$ \\
\hline Parkinson's disease sleep scale & $-0.01[-0.04,0.00]$ & 0.01 & 0.00 & -0.15 & 0.171 \\
\hline Epworth Sleepiness Scale & $0.15[0.01,0.34]$ & 0.08 & 0.00 & 0.25 & 0.015 \\
\hline \multicolumn{6}{|l|}{ Multiple regression $^{\mathrm{b}}$} \\
\hline Fatigue Severity Scale & $0.42[0.09,0.82]$ & 0.17 & 0.00 & 0.31 & 0.007 \\
\hline Beck Depression Inventory & $0.13[0.03,0.23]$ & 0.05 & 0.00 & 0.39 & 0.004 \\
\hline Parkinson Anxiety Scale & $0.00[-0.05,0.06]$ & 0.02 & -0.00 & 0.02 & 0.823 \\
\hline Apathy Evaluation Scale & $-0.01[-0.07,0.04]$ & 0.03 & 0.00 & -0.05 & 0.575 \\
\hline Epworth Sleepiness Scale & $0.00[-0.10,0.11]$ & 0.07 & 0.00 & 0.00 & 0.929 \\
\hline
\end{tabular}

Statistically significant variables are shown in bold

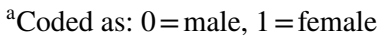

${ }^{\mathrm{b}}$ Model $(F$ test $)=10.89, p$ value $<0.001, R^{2}=0.42$

$S E$ standard error, $C I$ confidence interval, UPDRS Unified Parkinson's Disease Rating Scale, LEDD levodopa equivalent daily dose inflated the objective-subjective cognitive discrepancy. Indeed, the cognitive functions implied in daily activities, as explored by PD-CFRS, may not necessarily parallel those needed to perform a cognitive screening test. Consequently, patients classified as "Understimators" may be affected by an objective cognitive impairment undetected by MoCA. On the other hand, as MoCA is a cognitive screening test recommended by the MDS, widely used in clinical practice and research, this could help ensuring a reasonable generalization of our results $[11,20]$. Moreover, we considered MoCA subscores to increase the explanatory power of our results and to provide a more nuanced assessment of objective cognitive functioning [61].

An additional limitation of this study is its cross-sectional nature which calls for replication by longitudinal study designs, by which it is possible to explore the predictive relationship between the self-experienced worsening of cognitive capacities and the objective cognitive deterioration
[46]. Furthermore, to screen the presence of PD dementia, we used the level I testing procedures which, compared to level II ones, do not allow to specify the pattern and severity of the cognitive impairment and may lead to suboptimal recruitment decisions [28]. Finally, the low percentage of "Overestimators" did not enable us to use more powerful statistical methods, such as multinomial logistic regression analyses, to characterize the profile of this group.

Despite these shortcomings, our findings provide further insights on the main factors correlated with the discrepancy between objective and subjective cognitive functioning in PD. Accurate detection of cognitive impairment is crucial for guiding treatments and neuropsychological assessment in a patient-centered manner. This calls for a need of increased awareness of 'core' behavioral symptoms such as fatigue and depression, which are likely antecedents of the discrepancy between objective cognitive impairments and subjective cognitive complaints in PD. 
Fig. 2 A 3-D scatterplot showing the regression of MoCA $(Z$ score) minus PD-CFRS ( $Z$ score) on Beck Depression Inventory and Fatigue Severity Scale; the tinted trapezium (regression plane) is built by linear multiple regression equation and the dots represent the observed data points; the color bar represents the values of beta coefficients

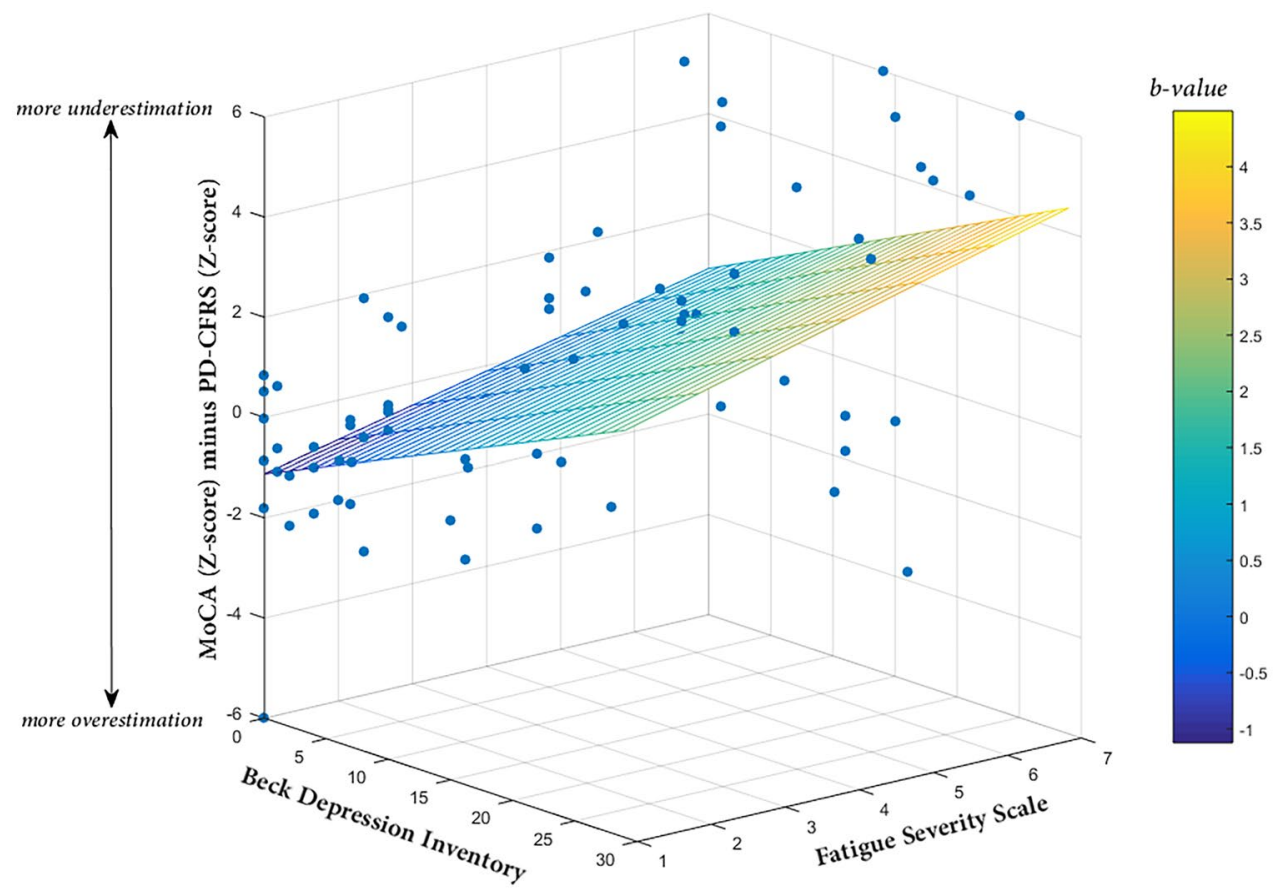

The further implications of our study are threefold. First, caution should be exercised when making a clinical diagnosis of MCI, especially when MCI is diagnosed only in terms of subjectively-reported cognitive complaints $[1,6]$. Second, clinicians should include an assessment of fatigue and depression in their routine cognitive examination to determine to extent to which these behavioral factors influence cognitive complains. Third, our data might suggest that behavioral and/or pharmacological interventions for fatigue and depression could reduce subjective cognitive complaints [62].

Enhancing patients' abilities to correctly perceive their individual level of cognitive functioning has great potential to improve their own and their caregivers' quality of life.

Supplementary Information The online version contains supplementary material available at https://doi.org/10.1007/s00415-021-10519-4.

Author contributions All authors contributed to the study conception and design. Material preparation, data collection and analysis were performed by MS, LT, and AT. The first draft of the manuscript was written by MS and all authors commented on previous versions of the manuscript. All authors read and approved the final manuscript.

Funding Luca Passamonti was funded by the Medical Research Council (MRC) (Grant Number MR/P01271X/1).

Data availability Research data were not shared.

\section{Declarations}

Conflicts of interest Not applicable.
Ethical approval Approval was obtained from the ethics committee of University of Campania "Luigi Vanvitelli" (Naples, Italy). The procedures used in this study adhere to the tenets of the Declaration of Helsinki.

Consent to participate Informed consent was obtained from all individual participants included in the study.

Consent for publication Patients signed informed consent regarding publishing their data.

Open Access This article is licensed under a Creative Commons Attribution 4.0 International License, which permits use, sharing, adaptation, distribution and reproduction in any medium or format, as long as you give appropriate credit to the original author(s) and the source, provide a link to the Creative Commons licence, and indicate if changes were made. The images or other third party material in this article are included in the article's Creative Commons licence, unless indicated otherwise in a credit line to the material. If material is not included in the article's Creative Commons licence and your intended use is not permitted by statutory regulation or exceeds the permitted use, you will need to obtain permission directly from the copyright holder. To view a copy of this licence, visit http://creativecommons.org/licenses/by/4.0/.

\section{References}

1. Litvan I, Goldman JG, Tröster AI, Schmand BA, Weintraub D, Petersen RC, Mollenhauer B, Adler CH, Marder K, WilliamsGray CH, Aarsland D, Kulisevsky J, Rodriguez-Oroz MC, Burn DJ, Barker RA, Emre M (2012) Diagnostic criteria for mild cognitive impairment in Parkinson's disease: Movement Disorder Society Task Force guidelines. Mov Disord 27:349-356

2. Bailey M, Goldman JG (2017) characterizing cognitive impairment in Parkinson's disease. Semin Neurol 37:167-175 
3. Baiano C, Barone P, Trojano L, Santangelo G (2020) Prevalence and clinical aspects of mild cognitive impairment in Parkinson's disease: a meta-analysis. Mov Disord 35:45-54

4. Yoo HS, Chung SJ, Lee YH, Ye BS, Sohn YH, Lee PH (2020) Cognitive anosognosia is associated with frontal dysfunction and lower depression in Parkinson's disease. Eur J Neurol 27:951-958

5. Koerts J, Tucha L, Leenders KL, van Beilen M, Brouwer WH, Tucha O (2011) Subjective and objective assessment of executive functions in Parkinson's disease. J Neurol Sci 310:172-175

6. Copeland JN, Lieberman A, Oravivattanakul S, Tröster AI (2016) Accuracy of patient and care partner identification of cognitive impairments in Parkinson's disease-mild cognitive impairment. Mov Disord 31:693-698

7. Dujardin K, Duhamel A, Delliaux M, Thomas-Antérion C, Destée A, Defebvre L (2009) Cognitive complaints in Parkinson's disease: its relationship with objective cognitive decline. J Neurol 257:79-84

8. Hong JY, Sunwoo MK, Chung SJ, Ham JH, Lee JE, Sohn YH, Lee PH (2014) Subjective cognitive decline predicts future deterioration in cognitively normal patients with Parkinson's disease. Neurobiol Aging 35:1739-1743

9. Lehrner J, Moser D, Klug S, Gleiß A, Auff E, Pirker W, Pusswald G (2014) Subjective memory complaints, depressive symptoms and cognition in Parkinson's disease patients. Eur J Neurol 21:1276-1284

10. Erro R, Santangelo G, Barone P, Picillo M, Amboni M, Longo K, Giordano F, Moccia M, Allocca R, Pellecchia MT, Vitale C (2014) Do subjective memory complaints herald the onset of mild cognitive impairment in Parkinson disease? J Geriatr Psychiatry Neurol 27:276-281

11. Mills KA, Mari Z, Pontone GM, Pantelyat A, Zhang A, Yoritomo N, Powers E, Brandt J, Dawson TM, Rosenthal LS (2016) Cognitive impairment in Parkinson's disease: association between patient-reported and clinically measured outcomes. Parkinsonism Relat Disord 33:107-114

12. Hong JY, Lee Y, Sunwoo MK, Sohn YH, Lee PH (2018) Subjective cognitive complaints and objective cognitive impairment in Parkinson's disease. J Clin Neurol 14:16-21

13. Galtier I, Nieto A, Lorenzo JN, Barroso J (2019) Subjective cognitive decline and progression to dementia in Parkinson's disease: a long-term follow-up study. J Neurol 266:745-754

14. Purri R, Brennan L, Rick J, Xie SX, Deck BL, Chahine LM, Dahodwala N, Chen-Plotkin A, Duda JE, Morley JF, Akhtar RS, Trojanowski JQ, Siderowf A, Weintraub D (2020) Subjective cognitive complaint in Parkinson's disease patients with normal cognition: canary in the coal mine? Mov Disord 35:1618-1625

15. Orfei MD, Assogna F, Pellicano C, Pontieri FE, Caltagirone C, Pierantozzi M, Stefani A, Spalletta G (2018) Anosognosia for cognitive and behavioral symptoms in Parkinson's disease with mild dementia and mild cognitive impairment: Frequency and neuropsychological/neuropsychiatric correlates. Parkinsonism Relat Disord 54:62-67

16. Rosen HJ (2011) Anosognosia in neurodegenerative disease. Neurocase 17:231-241

17. Kjeldsen PL, Damholdt MF (2019) Subjective cognitive complaints in patients with Parkinson's disease. Acta Neurol Scand 140:375-389

18. Sitek EJ, Sołtan W, Wieczorek D, Robowski P, Sławek J (2011) Self-awareness of memory function in Parkinson's disease in relation to mood and symptom severity. Aging Ment Health $15: 150-156$

19. Dupouy J, Ory-Magne F, Mekies C, Rousseau V, Puel M, Rerat K, Pariente J, Brefel-Courbon C, PARKMIP group, (2018) Cognitive complaint in early Parkinson's disease: a pilot study. Acta Neurol Scand 137:59-66
20. Mills KA, Schneider RB, Saint-Hilaire M, Ross GW, Hauser RA, Lang AE, Halverson MJ, Oakes D, Eberly S, Litvan I, Blindauer K, Aquino C, Simuni T, Marras C (2020) Cognitive impairment in Parkinson's disease: Associations between subjective and objective cognitive decline in a large longitudinal study. Parkinsonism Relat Disord 80:127-132

21. Koster DP, Higginson CI, MacDougall EE, Wheelock VL, Sigvardt KA (2015) subjective cognitive complaints in Parkinson disease without dementia: a preliminary study. Appl Neuropsychol Adult 22:287-292

22. Siciliano M, Trojano L, De Micco R, Russo A, Tedeschi G, Tessitore A (2020) Subjective memory decline in Parkinson's disease patients with and without fatigue. Parkinsonism Relat Disord 70:15-19

23. Tsapanou A, Vlachos GS, Cosentino S, Gu Y, Manly JJ, Brickman AM, Schupf N, Zimmerman ME, Yannakoulia M, Kosmidis MH, Dardiotis E, Hadjigeorgiou G, Sakka P, Stern Y, Scarmeas N, Mayeux R (2019) Sleep and subjective cognitive decline in cognitively healthy elderly: results from two cohorts. J Sleep Res 28:e12759

24. De Carolis A, Cipollini V, Corigliano V, Comparelli A, SepeMonti M, Orzi F, Ferracuti S, Giubilei F (2015) Anosognosia in people with cognitive impairment: association with cognitive deficits and behavioral disturbances. Dement Geriatr Cogn Disord Extra 5:42-50

25. Valentino V, Iavarone A, Amboni M, Moschiano F, Picillo M, Petretta V, Cicarelli G (2018) Apathy in Parkinson's disease: differences between caregiver's report and self-evaluation. Funct Neurol 33:31-35

26. Santangelo G, Vitale C, Trojano L, Angrisano MG, Picillo M, Errico D, Agosti V, Grossi D, Barone P (2014) Subthreshold depression and subjective cognitive complaints in Parkinson's disease. Eur J Neurol 21:541-544

27. Sheehan DV, Lecrubier Y, Sheehan KH, Amorim P, Janavs J, Weiller E, Hergueta T, Baker R, Dunbar GC (1998) The MiniInternational Neuropsychiatric Interview (M.I.N.I.): the development and validation of a structured diagnostic psychiatric interview for DSM-IV and ICD-10. J Clin Psychiatry 59(Suppl 20):22-33

28. Dubois B, Burn D, Goetz C, Aarsland D, Brown RG, Broe GA, Dickson D, Duyckaerts C, Cummings J, Gauthier S, Korczyn A, Lees A, Levy R, Litvan I, Mizuno Y, McKeith IG, Olanow CW, Poewe W, Sampaio C, Tolosa E, Emre M (2007) Diagnostic procedures for Parkinson's disease dementia: recommendations from the movement disorder society task force. Mov Disord 22:2314-2324

29. Santangelo G, Siciliano M, Pedone R, Vitale C, Falco F, Bisogno R, Siano P, Barone P, Grossi D, Santangelo F, Trojano L (2015) Normative data for the Montreal Cognitive Assessment in an Italian population sample. Neurol Sci 36:585-591

30. Fahn S, Elton RL, Development Committee UPDRS (1987) The unified Parkinson's disease rating scale. In: Fahn S, Marsden CD, Calne DB, Goldstein M (eds) Recent developments in Parkinson's disease, 2nd edn. Macmillan Healthcare Information, New Jersey

31. Goetz CG, Poewe W, Rascol O, Sampaio C, Stebbins GT, Counsell C, Giladi N, Holloway RG, Moore CG, Wenning GK, Yahr MD, Seidl L, Movement Disorder Society Task Force on Rating Scales for Parkinson's Disease (2004) Movement Disorder Society Task Force report on the Hoehn and Yahr staging scale: status and recommendations. Mov Disord 19:1020-1028

32. Tomlinson CL, Stowe R, Patel S, Rick C, Gray R, Clarke CE (2010) Systematic review of levodopa dose equivalency reporting in Parkinson's disease. Mov Disord 15:2649-2653

33. Krupp LB, LaRocca NG, Muir-Nash J, Steinberg AD (1989) The fatigue severity scale. Application to patients with 
multiple sclerosis and systemic lupus erythematosus. Arch Neurol 46:1121-1123

34. Siciliano M, Chiorri C, De Micco R, Russo A, Tedeschi G, Trojano L, Tessitore A (2019) Fatigue in Parkinson's disease: Italian validation of the Parkinson Fatigue Scale and the Fatigue Severity Scale using a Rasch analysis approach. Parkinsonism Relat Disord 65:105-110

35. Leentjens AF, Verhey FR, Luijckx GJ, Troost J (2000) The validity of the Beck Depression Inventory as a screening and diagnostic instrument for depression in patients with Parkinson's disease. Mov Disord 15:1221-1224

36. Leentjens AF, Dujardin K, Pontone GM, Starkstein SE, Weintraub D, Martinez-Martin P (2014) The Parkinson Anxiety Scale (PAS): development and validation of a new anxiety scale. Mov Disord 29:1035-1043

37. Santangelo G, Falco F, D'Iorio A, Cuoco S, Raimo S, Amboni M, Pellecchia MT, Longo K, Vitale C, Barone P (2016) Anxiety in early Parkinson's disease: validation of the Italian observer-rated version of the Parkinson Anxiety Scale (OR-PAS). J Neurol Sci 367:158-161

38. Marin RS, Biedrzycki RC, Firinciogullari S (1991) Reliability and validity of the Apathy Evaluation Scale. Psychiatry Res $38: 143-162$

39. Santangelo G, Barone P, Cuoco S, Raimo S, Pezzella D, Picillo M, Erro R, Moccia M, Pellecchia MT, Amboni M, Santangelo F, Grossi D, Trojano L, Vitale C (2014) Apathy in untreated, de novo patients with Parkinson's disease: validation study of Apathy Evaluation Scale. J Neurol 261:2319-2328

40. Johns MW (1991) A new method for measuring daytime sleepiness: the Epworth sleepiness scale. Sleep 14:540-545

41. Chaudhuri KR, Pal S, Di Marco A, Whately-Smith C, Bridgman K, Mathew R, Pezzela F, Forbes A, Hogl B, Trenkwalder C (2002) The Parkinson's disease sleep scale: a new instrument for assessing sleep and nocturnal disability in Parkinson's disease. J Neurol Neurosurg Psychiatry 73:629-663

42. Nasreddine ZS, Phillips NA, Bédirian V, Charbonneau S, Whitehead V, Collin I, Cummings JL, Chertkow H (2005) The Montreal Cognitive Assessment, MoCA: a brief screening tool for mild cognitive impairment. J Am Geriatr Soc 53:695-699

43. Kulisevsky J, Fernández de Bobadilla R, Pagonabarraga J, Martínez-Horta S, Campolongo A, García-Sánchez C, Pascual-Sedano B, Ribosa-Nogué R, Villa-Bonomo C (2013) Measuring functional impact of cognitive impairment: validation of the Parkinson's disease cognitive functional rating scale. Parkinsonism Relat Disord 19:812-817

44. Santangelo G, Cuoco S, Barone P (2015) Parkinson's disease Cognitive Functional Rating Scale, Italian version. https://www. movementscales.com/formulario-func-italian

45. Ruzafa-Valiente E, Fernández-Bobadilla R, García-Sánchez C, Pagonabarraga J, Martínez-Horta S, Kulisevsky J (2016) Parkinson's disease-Cognitive Functional Rating Scale across different conditions and degrees of cognitive impairment. J Neurol Sci 361:66-71

46. Jessen F, Amariglio RE, van Boxtel M, Breteler M, Ceccaldi M, Chételat G, Dubois B, Dufouil C, Ellis KA, van der Flier WM, Glodzik L, van Harten AC, de Leon MJ, McHugh P, Mielke MM, Molinuevo JL, Mosconi L, Osorio RS, Perrotin A, Petersen RC, Rabin LA, Rami L, Reisberg B, Rentz DM, Sachdev PS, de la Sayette V, Saykin AJ, Scheltens P, Shulman MB, Slavin MJ, Sperling RA, Stewart R, Uspenskaya O, Vellas B, Visser PJ, Wagner
M, Subjective Cognitive Decline Initiative (SCD-I) Working Group (2014) A conceptual framework for research on subjective cognitive decline in preclinical Alzheimer's disease. Alzheimers Dement 10:844-852

47. Landis JR, Koch GG (1977) The measurement of observer agreement for categorical data. Biometrics 33:159-174

48. Hughes AJ, Bhattarai JJ, Paul S, Beier M (2019) Depressive symptoms and fatigue as predictors of objective-subjective discrepancies in cognitive function in multiple sclerosis. Mult Scler Relat Disord 30:192-197

49. Efron B, Tibshirani RJ (1993) An introduction to the bootstrap. Chapman and Hall, New York

50. Kinsinger SW, Lattie E, Mohr DC (2010) Relationship between depression, fatigue, subjective cognitive impairment, and objective neuropsychological functioning in patients with multiple sclerosis. Neuropsychology 24:573-580

51. Kluger BM, Herlofson K, Chou KL, Lou JS, Goetz CG, Lang AE, Weintraub D, Friedman J (2016) Parkinson's disease-related fatigue: a case definition and recommendations for clinical research. Mov Disord 31:625-631

52. Kluger BM, Krupp LB, Enoka RM (2013) Fatigue and fatigability in neurologic illnesses: proposal for a unified taxonomy. Neurology 80:409-416

53. Kuppuswamy A (2017) The fatigue conundrum. Brain 140:2240-2245

54. Petersen JZ, Porter RJ, Miskowiak KW (2019) Clinical characteristics associated with the discrepancy between subjective and objective cognitive impairment in depression. J Affect Disord 246:763-774

55. Hoshi Y, Oda I, Wada Y, Ito Y, Yamashita Y, Oda M, Ohta K, Yamada Y, Mamoru T (2000) Visuospatial imagery is a fruitful strategy for the digit span backward task: a study with near-infrared optical tomography. Brain Res Cogn Brain Res 9:339-342

56. Rueckert L, Lange N, Partiot A, Appollonio I, Litvan I, Le Bihan D, Grafman J (1996) Visualizing cortical activation during mental calculation with functional MRI. Neuroimage 3:97-103

57. Kudlicka A, Clare L, Hindle J (2013) Awareness of executive deficits in people with Parkinson's disease. J Int Neuropsychol Soc 19:559-570

58. Hannesdottir K, Morris RG (2007) Primary and secondary anosognosia for memory impairment in patients with Alzheimer's disease. Cortex 43:1020-1030

59. Zbozinek TD, Rose RD, Wolitzky-Taylor KB, Sherbourne C, Sullivan G, Stein MB, Roy-Byrne PP, Craske MG (2012) Diagnostic overlap of generalized anxiety disorder and major depressive disorder in a primary care sample. Depress Anxiety 29:1065-1071

60. Eysenck MW, Fajkowska M (2018) Anxiety and depression: toward overlapping and distinctive features. Cogn Emot 32:1391-1400

61. Vogel SJ, Banks SJ, Cummings JL, Miller JB (2015) Concordance of the Montreal cognitive assessment with standard neuropsychological measures. Alzheimers Dement 1:289-294

62. Seppi K, Ray Chaudhuri K, Coelho M, Fox SH, Katzenschlager R, Perez Lloret S, Weintraub D, Sampaio C, the collaborators of the Parkinson's Disease Update on Non-Motor Symptoms Study Group on behalf of the Movement Disorders Society EvidenceBased Medicine Committee (2019) Update on treatments for nonmotor symptoms of Parkinson's disease-an evidence-based medicine review. Mov Disord 34:180-198 